\title{
Comet interceptor's EnVisS camera sky mapping function
}

Pernechele, Claudio, Da Deppo, Vania, Brydon, George, Jones, Geraint, Lara, Luisa, et al.

Claudio Pernechele, Vania Da Deppo, George Brydon, Geraint H. Jones, Luisa Lara, Harald Michaelis, "Comet interceptor's EnVisS camera sky mapping function," Proc. SPIE 11203, Advances in Optical Astronomical Instrumentation 2019, 112031M (3 January 2020); doi: 10.1117/12.2539239 


\title{
Comet Interceptor's EnVisS Camera Sky Mapping Function
}

\author{
Claudio Pernechele ${ }^{* a}$, Vania Da Deppo ${ }^{\mathrm{b}}$, George Brydon ${ }^{\mathrm{c}, \mathrm{d}}$, \\ Geraint H. Jones ${ }^{\mathrm{c}, \mathrm{d}}$, Luisa Lara ${ }^{\mathrm{e}}$, Harald Michaelis ${ }^{\mathrm{f}}$ \\ ${ }^{a}$ National Institute for Astrophysics (INAF), Vicolo Osservatorio 5, 35122 Padova, IT; \\ ${ }^{\mathrm{b}}$ CNR-IFN Padova, Via Trasea 7, 35131 Padova, IT; \\ ${ }^{c}$ UCL Mullard Space Science Laboratory, Holmbury St. Mary, Surrey RH5 6NT, UK; \\ ${ }^{\mathrm{d}}$ Centre for planetary Science @UCL/Birkbeck, Gover Street, London, WC1E 6NT, UK; \\ eInstituto de Astrofisica de Andalucia - CSIC, Apartado 3004, 18080 Granada, ES; \\ ${ }^{\mathrm{f}}$ DLR Institute of Planetary Research, Rutherfordstraße 2, 12489, Berlin, DE.
}

\begin{abstract}
Entire Visible Sky (EnVisS) is one of the payload proposed for the ESA selected F-Class mission Comet Interceptor. The main aim of the mission is the study of a dynamically new comet, or an interstellar object, entering the inner solar system.

The EnVisS camera is designed to capture the entire sky in some visible wavelength bands while the spacecraft passes through the comet's tail environment. EnVisS optical head is composed of a fisheye lens with a field of view of $180^{\circ} \mathrm{x}$ $40^{\circ}$ coupled with an imaging detector equipped with both band-pass and polarimetric filters.

Very wide angle lens, as a fisheye, must be necessarily anamorphic, i. e. its focal length must change along the field of view, in order to fit a finite-size imaging detector. This anamorphic distortion is introduced by the optical designer, depending on the desired applications. Each possible distortion bring along different field of view mapping and this must be taken into account by the scientific/metrological user, because the plate scale is variable along the focal plane.

To obtain useful scientific information from fisheye images (astrometry, flux calibration and brightness measurements), a precise determination of the mapping function has to be accurately determined. In this paper we describe the expected distortion map of the EnVisS camera.
\end{abstract}

Keywords: fisheye lenses, wide angle lenses, lens mapping function, astronomical imaging.

\section{INTRODUCTION}

Fisheye is the paradigm of a very wide angle lens. Despite their quite small entrance pupil, very wide angle lenses found some applications in astronomical research. These lenses have been proposed for space-based observations of heliospheric features [1]; the Solar Mass Ejection Imager (SMEI) aboard the Coriolis spacecraft has been designed to detect and forecast the arrival of solar mass ejections [2]; the Mars Color Imager (MARCI) aboard the Mars Reconnaissance Orbiter spacecraft [3] use a fisheye optics; an explorative study for applying hyper hemispheric lens as a medium-resolution star tracker for mini and micro-satellites has been published [4,5]; in ground based astronomy, fisheye is used in all-sky photographic cameras to determine meteor trajectory [6]. In astronomical or, more generally, metrological application of very wide angle lenses, an accurate determination on how the space is mapped into the focal plane is mandatory to obtain calibrated data, both for real/apparent orbit determination (astrometry) and flux/brightness calibration. Each application may need a different mapping function and the optical designer should bear this in mind in optimizing the proper fisheye layout. We describe here different possible sky mapping functions, with particular regard to the Comet Interceptor EnVisS camera [7].

\section{FISHEYE MAPPING FUNCTIONS}

The design of very wide angle lenses requires to account for some typical issues. The fundamental origin of the optical problems resides on the entrance pupil shift at large angles, where the paraxial approximation is no more valid: chief rays angles on the object side are not preserved passing through the optics preceding the aperture stop (fore-optics). This

Advances in Optical Astronomical Instrumentation 2019, edited by Simon Ellis, Céline d'Orgeville, Proc. of SPIE Vol. 11203, 112031M · @ 2020 SPIE · CCC code: 0277-786X/20/\$21 · doi: 10.1117/12.2539239 
effect produces an anamorphic deformation on the focal plane, with the focal length changing along the elevation angles. With reference to the Fig. 1, when the zenith (or field) angle $\mathrm{Z}$ becomes wide, the aperture stop is seen slanting and, at the limit angle of $90^{\circ}$, it will result completely obstructed. In order to permit the visibility of objects at high $\mathrm{Z}$ fields, the entrance pupil should be tilted. This movement is possible at the cost of some pupil compression, which alters the exit pupil flux.

Referring to Fig. 1, the "perfect" undistorted map of the object space is one where $\psi=Z$, i.e. $\mathrm{R}=f \cdot \tan (\mathrm{Z})$ with $f$ the paraxial focal length. Every point in the space is mapped maintaining the same angular distribution into the focal plane

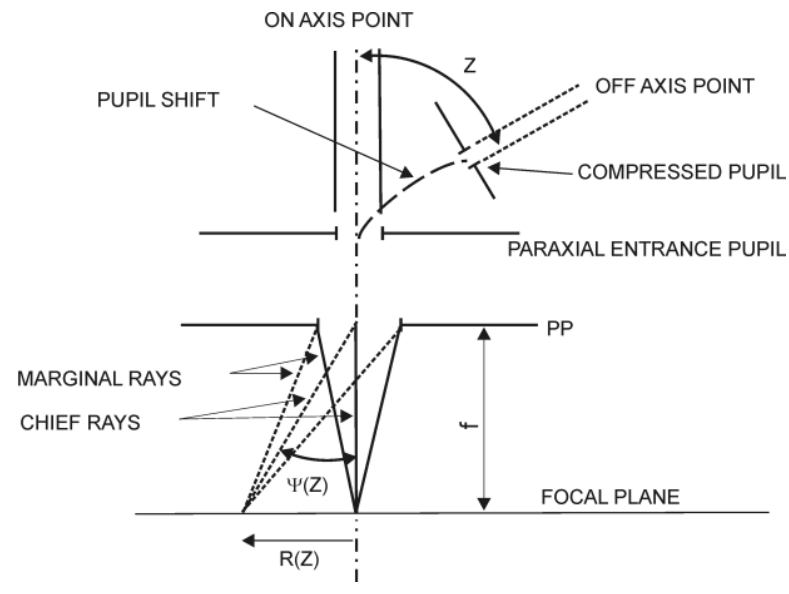

Fig 1: Image formation in a very wide angle lens.

and object straight lines remain straight (distortion free) in the image. This function is known as perspective projection and has no meaning for wide zenith angles, because the focal plane would be infinitely wide and the entrance pupil (the pin-hole) would be completely obscured at $Z=90^{\circ}$. The function is shown as a dotted line in the Fig. 2 , where normalized $\mathrm{R} / f$ coordinates are plotted versus $Z$. In order to have a wide-angle lens useful for any application, some image distortion has to be introduced. Equidistant (linear scaled) projection has the form $\mathrm{R}=f$. $\mathrm{Z}$. The equidistant function maintains angular distances and it is traced as a dashed line in Fig. 2. The most generic mapping function is defined as:

$$
R=f \cdot k_{1} \cdot \sin \left(k_{2} \cdot Z\right),
$$

with $k_{1}$ and $k_{2}$ adimensional coefficients. One traditional and useful projection scheme is the equisolid angle projection (with $k_{1}=2, k_{2}=0.5$ ), which maintains surface relations. Each pixel in the detector subtends an equal solid angle, i.e. an equal area on the unit sphere. Its mapping function is traced as long-dashed line in Fig. 2. In the orthographic projection,

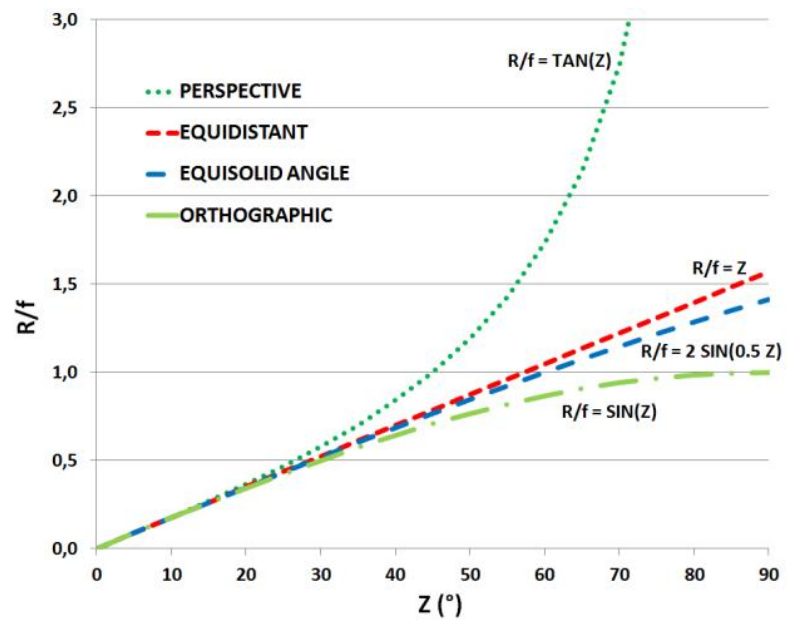

Fig 2: Fisheye common mapping functions. 
also known as sine-law projection $\left(k_{l}=k_{2}=1\right)$, the focal plane radial distribution is $\mathrm{R}=\mathrm{f} \cdot \sin (\mathrm{Z})$. This projection maintains planar illuminance and the marginal fields are extremely compressed at the focal plane. Its mapping function is plotted as dot-dashed line in Fig. 2.

\subsection{Fisheye plate scale}

Due to the high distortion at very wide angles, the spatial resolution of an object changes with respect to zenith angles $\mathrm{Z}$. Inverting the generic projection map [1], we can express the zenith angles $Z$ as a function of the focal plane radial coordinate $\mathrm{Z}=\mathrm{Z}(\mathrm{R})$ and the plate scale along $\mathrm{Z}$ becomes the differential of the relation [1]:

$$
R E S_{Z}(R)=\frac{\partial Z}{\partial R}=\frac{1}{f} \frac{1}{k_{1} \cdot k_{2} \sqrt{1-\left[R /\left(d \cdot k_{1}\right)\right]^{2}}} \mathrm{~mm}^{-1}
$$

The angular resolution along $\mathrm{Z}$ decreases at higher zenith angle, as expected. For the calculation of the plate scale along the azimuth axis we have to consider that $A Z(Z)=2 \pi \cdot \sin (Z)$ (see left panel of Fig. 3). For example, $A Z=360^{\circ}$ when $\mathrm{Z}=90^{\circ}$ (at the lens horizon). The $\mathrm{AZ}(\mathrm{Z})$ projection onto the focal plane is a circumference of length $2 \pi \cdot \mathrm{R}(\mathrm{Z})$ (see right panel of Fig. 3) and then the plate scale along the Azimuth become

$$
R E S_{A Z}(R)=\frac{A Z}{2 \pi R(Z)}=\frac{\sin (Z)}{R(Z)} \mathrm{mm}^{-1}
$$

The difference in values between the spatial resolution along the zenith $(Z)$ and azimuth $(A Z)$ axes reflects the anamorphism of the fisheye lens.

\subsection{EnVisS plate scale}

The EnVisS camera will map a sky belt of $180^{\circ} \mathrm{x} 40^{\circ}$ centered on the lens zenith (left panel in Fig. 3).
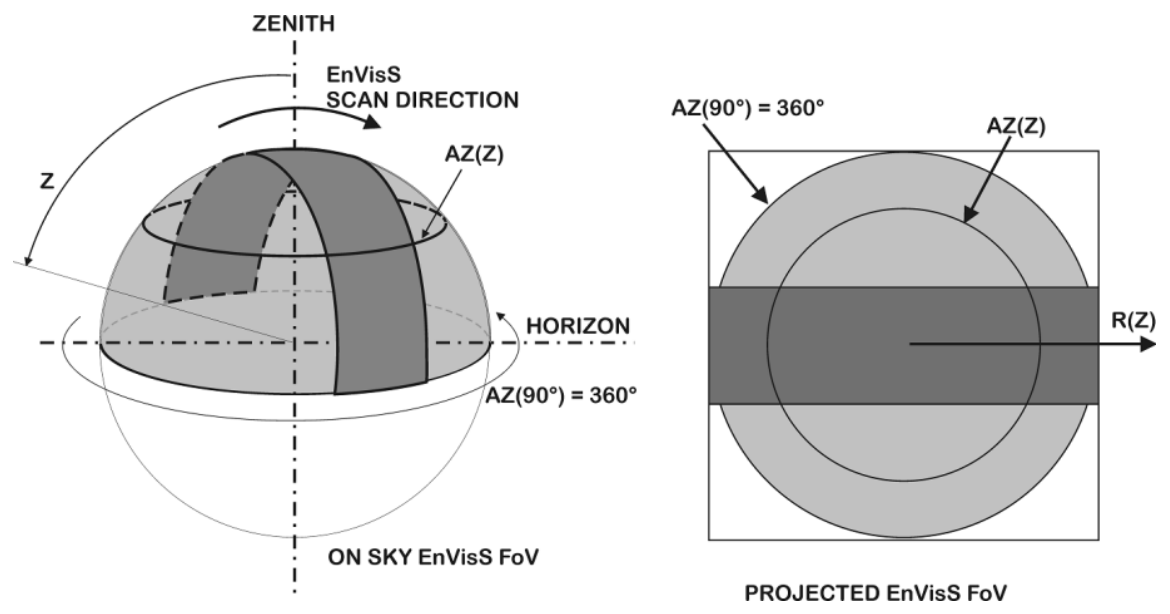

Fig 3: EnVisS field of view in the real-space (left panel) and the focal plane (right panel).

Choosing for it an equidistant linear-scaled projection scheme $(\mathrm{R} / f=\mathrm{Z})$, the plate scales on the focal plane become:

$$
\begin{gathered}
R E S_{Z}=\frac{1}{f} \mathrm{~mm}^{-1} \\
R E S_{A Z}=\frac{1}{f} \frac{\sin (Z)}{Z} \mathrm{~mm}^{-1}
\end{gathered}
$$


The resolution is constant along the elevation axis (by construction), while along the AZ axis it increase with $\mathrm{Z}$. The normalized AZ resolution is plotted in Fig. 4, and, as expected, the resolution becomes finer as $\mathrm{Z}$ increases, i. e. the length of the projected circumference of the on-sky azimuth circles (see Fig. 3) grows faster than the AZ(Z) values.

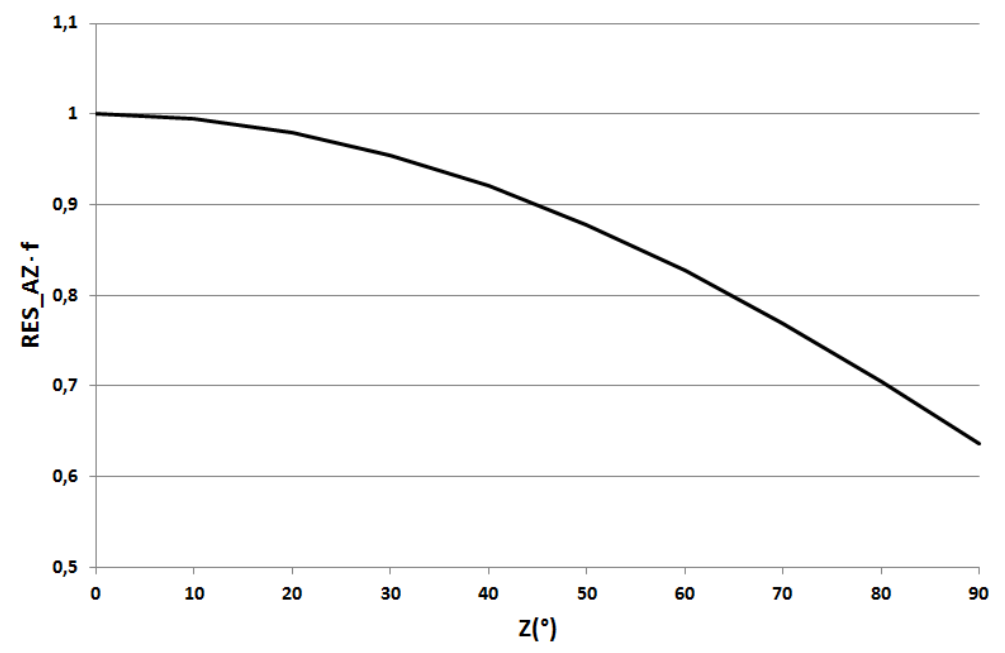

Fig 4: The $\mathrm{AZ}$ resolution increases with $\mathrm{Z}$ (The $\mathrm{Z}$ resolution remain equal to the unity at every $\mathrm{Z}$ values).

The instantaneous field view as viewed from a detector pixel is:

$$
I F o V=\frac{1}{f} \Delta p x \cdot \frac{1}{f} \frac{\sin (Z)}{Z} \Delta p x
$$

with $\Delta p x$ the (squared) pixel dimension and $f$ the lens paraxial focal length. The multiplication factors are maintained separate to underline the lens anamorphism. Note as the instantaneous field of view is different along the $\mathrm{Z}$ (the former factor, independent by the $\mathrm{Z}$ angles due to the lens's equidistant projection design) and $\mathrm{AZ}$ (the second one, variable with $\mathrm{Z})$ axes. In particular, being $[\sin ((\mathrm{Z}) / \mathrm{Z}] \leq 1$, the pixel resolution along $\mathrm{AZ}$ become finest approaching the horizon.

\section{REFERENCES}

[1] Buffington, A., et al., "Corrals, hubcaps and crystal balls: some new design for very-wide-angle visible-light heliospheric imagers", proc. SPIE 3442, 77-86 (1998).

[2] Eyles, C. J., et al., "The Solar Mass Ejection Imager (SMEI)", Solar Physics, vol. 217, 319-347 (2003).

[3] Bell III, J. F., et al., "Mars Reconnaissance Orbiter Mars Color Imager (MARCI): Instrument description, calibration and performance", Journ. of Geophys. Res., vol. 114, doi:10.1029/2008JE003315 (2009).

[4] Pernechele, C., et al., " Hyper hemispheric lens applications in small and micro satellites", Adv. in Space Science, doi: 10.1016/j.asr.2018.02.025 (2018).

[5] Opromolla, R, et al., " A new star tracker concept for satellite attitude determination based on a multi-purpose panoramic camera", Acta Astronautica, doi: 10.1016/j.actaastro.2017.08.020 (2017).

[6] Borovička, J., Spurný, P., Keclíková, J, "A new positional astrometric method for all-sky cameras", A\&ASS, 112, 173-178 (1995).

[7] Brydon, G., Jones, G. H., "Comet Interceptor's EnVisS Camera: Multispectral and Polarimetric Full-sky Imager for a Comet Flyby", EPSC-DPS Meeting, Vol. 13, EPCS-DPS2019-1691-1 (2019). 